\title{
PERCEIVED RISK AND ABILITIES FOR HEALTH PRACTICES RELATED TO PREVENTION OF TYPE II DIABETES MELLITUS AMONG POSTNATAL MOTHERS WITH GESTATIONAL DIABETES MELLITUS
}

\author{
BENCY BHASY, LINDA VARGHESE*, ASWATHY KRISHNAN, LEKHA VISWANATH \\ Department of OBG Nursing, Amrita College of Nursing, Amrita Vishwa Vidyapeetham, Kochi, Kerala, India. \\ Email: bencybhasy991@gmail.com
}

Received: 27 June 2017, Revised and Accepted: 12 December 2017

\begin{abstract}
Objectives: The study is aimed to assess the perceived risk and abilities for health practices related to prevention of Type II diabetes mellitus (DM) among postnatal mothers with gestational DM (GDM).

Methods: This is a descriptive study involving 60 postnatal mothers at Amrita Institute of Medical Sciences, Kochi. Non-probability convenience sampling technique was used for the study, data collected through semi-structured questionnaire, risk perception survey for developing diabetes is used to assess the perceived risks and self-rated abilities for health practice scale is used to assess abilities for health practices.
\end{abstract}

Result: Most of the postnatal mothers 55\% with GDM had an average personal control and 53\% of postnatal mother with GDM were high optimistic that they will not develop Type II DM. In case of diabetes risk knowledge, more than half of the mothers 54\% with GDM had average perception. Based on health practices, majority of the postnatal mothers $63 \%$ with GDM have average abilities for health practices related to prevention of Type II DM.

Conclusion: Strategies should be designed to care for the postnatal mothers with GDM such as early detection and postpartum screening are mandatory to detect future DM.

Keywords: Perceived risks, Gestational diabetes mellitus, Postnatal mothers, Type II diabetes mellitus.

(C) 2018 The Authors. Published by Innovare Academic Sciences Pvt Ltd. This is an open access article under the CC BY license (http://creativecommons. org/licenses/by/4. 0/) DOI: http://dx.doi.org/10.22159/ajpcr.2018.v11i3.20991

\section{INTRODUCTION}

Gestational diabetes mellitus (GDM) is the single strongest population predictor of Type II DM development, and there is at least sevenfold increase in Type II DM in women who have had GDM compared to those with euglycemic pregnancies [1,3]. Many women with a diagnosis of GDM were unaware of the future risk of DM, while others simply do not engage in healthy lifestyle behaviors after delivery. Most of the women have misconception that, GDM only affects them during pregnancy and once the baby is delivered, the complication is no longer a health threat. Numerous research studies in the general population have identified that adoption of a healthy lifestyle such as healthy diet, exercise, and weight loss can prevent GDM, however, there are limited researchers who can focus on usual healthy practices in women with GDM [4]. Most researchers have focused on glycemic control [5] throughout the pregnancy for positive maternal and fetal outcomes, but management has been lacking after childbirth to prevent or delay the development of DM in childbearing women [6]. Hence, the researchers decided to assess the perceived risk and abilities for health practices related to prevention of Type II DM among postnatal mothers with GDM.

\section{Subjects and methods}

Data collection was preceded with 60 postnatal mothers with GDM. Non-probability convenience sampling technique was used for the study. After establishing rapport with the mothers, the importance of the study and procedures involved in the data collection was explained, and informed consent was taken before data collection. Subjects who met the inclusion criteria were taken. Subjects were taken approximately $25 \mathrm{~min}$ to complete the questionnaire. Semi-structured questionnaire is used to assess sociodemographic and clinical variables, risk perception survey for developing diabetes (RPS-DD) to assess perceived risk and self-rated abilities for health practice scale to assess health practices.

\section{RESULTS}

Sociodemographic variables were represented in Table 1.

Based on clinical variables, in terms of parity $53 \%$ of the postnatal mothers were second gravida and $30 \%$ of them were grand-multiparous mothers. Majority of the deliveries $(60 \%)$ were after 37 weeks of gestation. Regarding mode of delivery, $70 \%$ of the mothers undergone LSCS and 58\% were detected GDM during 28-36 weeks. Based on the treatment taken during the pregnancy, $53 \%$ of the mothers were taken insulin [7]. Majority of the mothers (53\%) have reported that they had a history of abortion and $30 \%$ of them had PCOD [8]. In case of family history, $80 \%$ of the postnatal mothers have a family history of DM. Majority of them (76\%) had delivered babies with birth weight $>3 \mathrm{~kg}$.

Regarding perceived risk, most of the postnatal mothers 55\% with GDM have an average personal control to prevent the development of Type II DM and 53\% of postnatal mother with GDM were high optimistic that they will not develop Type II DM. More than half of them 54\% have an average perception about diabetes risk knowledge. When the individual items of Diabetes Risk knowledge are considered, 53\% of the mothers give correct response in the item having a blood relative with diabetes and $42 \%$ of them have knowledge about having had diabetes during pregnancy will develop Type II DM. High scorers 60\% of postnatal mother with GDM have greater belief that health behaviors have benefits for the prevention of Type II DM and lower scorers $40 \%$ reflects greater barriers to lifestyle modification for the prevention of developing Type II DM. More than half $55 \%$ of the subjects perceived the risk that they have a slight chance and $63 \%$ with GDM perceive that changing lifestyle behaviors have moderate chance to reduce the risk for getting diabetes over the next 10 years. Majority (70\%) of the postnatal mothers with GDM perceived that recently made change in lifestyle behaviors will reduce the chance of getting Type II DM. 
Table 1: Distribution of the postnatal mothers with GDM based on clinical data $n=60$

\begin{tabular}{ll}
\hline Demographic variables & Frequency (\%) \\
\hline Age in years & \\
18-25 & $16(27)$ \\
$26-33$ & $35(58)$ \\
$34-41$ & $9(15)$ \\
Education & \\
Higher secondary & $18(30)$ \\
Graduates & $36(60)$ \\
Postgraduates & $6(10)$ \\
Occupation & \\
Employed & $38(63)$ \\
Unemployed & $22(37)$ \\
Place of residence & \\
Rural & $42(70)$ \\
Urban & $18(30)$ \\
Source of information & $15(25)$ \\
Internet/magazine & $10(17)$ \\
Health workers & $35(58)$ \\
Nil &
\end{tabular}

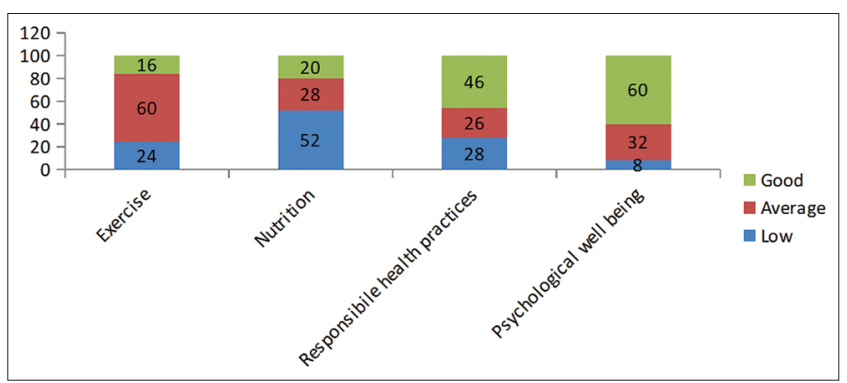

Fig. 1: Distribution of postnatal mothers with gestational diabetes mellitus based on the components of abilities for health practices related to prevention of Type II DM

Based on health practices, majority of the postnatal mothers $63 \%$ with GDM have average abilities for health practices related to prevention of Type II DM. Individual item of health practices is explained in Fig. 1.

Fig. 1 shows that, among specific components of abilities for health practices, majority of the postnatal mothers $60 \%$ got a high score in the component of psychological well-being. Among these mothers average score is more in the component of exercise $60 \%$ and low score is more in nutrition $52 \%$.

\section{DISCUSSION}

A randomized controlled trial study conducted by Zera et al., [9] about diabetes risk perception in women with recent GDM (2012) among 70 third trimester mothers. Risk perception for Type II DM was assessed using a questionnaire adapted from the RPS for DD. The result showed that women who perceived themselves to be at low personal control and had a greater optimistic bias for not developing Diabetes than women who perceived themselves to be at high risk (mean optimistic bias score $2.3 \pm 0.4$ vs. $1.9 \pm 0.3, p=0.0005)$. Knowledge of risk factors for diabetes overall was low (mean \pm SD score $6.0 \pm 1.9$, of 11 possible points), $85 \%$ of participants were aware that regular physical activity, controlling weight gain, and eating a healthy diet were behaviors that could decrease risk for Type II DM. Nearly $94 \%$ of the participants correctly identified GDM as a risk factor for future Type II DM and were aware of at least one preventive behavior that could decrease the risk of Type II DM. However, in the present study, 54\%of mothers have average personal control for the prevention of future Type II DM and majority 53\% of them were high optimistic that they will not develop Type II DM. Knowledge of risk factors for diabetes overall was average (mean \pm SD score $9.0 \pm 1.68$ ). Majority of the participants were not known that regular physical activity, controlling weight gain, and eating a healthy diet were behaviors that could decrease risk for Type II DM.

A study conducted by Amason [10], about the effect of an educational intervention in women with GDM among 223 postnatal mothers. Selfrated abilities for health practices scale were used to assess the health practices of mothers with GDM. The result showed that 95\% the mothers have low health practices. When each subscale was analyzed, mean score of psychological well-being 3.22 was higher than other subscales. Low mean score in the area of nutrition 2.97. In the present study majority, $56 \%$ of the postnatal mothers with GDM have average abilities to adopt health practices related to prevention of Type II DM. Among specific components of abilities for health practices, the high mean score $(9.3 \pm 2.66)$ were obtained in the area of psychological wellbeing. Average mean score $5.6 \pm 3.1$ were obtained from exercise. Low mean score is more in nutrition. While comparing these two result, both had obtain least score in the component of nutrition, which shows the mothers did not maintain adequate health practices in the component of nutrition such as maintaining balanced diet, having fiber-rich contain foods and they did not know that maintaining good nutrition will reduce the risk of developing DM.

The present study discussed with the review shows that majority of the mothers have average risk of Type II DM but based on their health practices the mothers have low practices.

\section{CONCLUSION}

The findings of the study showed that mothers had average risk perception and their usual health practices were average to prevent future Type II DM. Hence, strategies should be designed to care for the postnatal mothers with GDM and early detection, and postpartum screening test is mandatory to detect future DM.

\section{LIMITATIONS}

The study setting was confined to Amrita Institute of Medical Sciences, Kochi; therefore, the generalization of the findings was limited.

\section{FINANCIAL SUPPORT AND SPONSORSHIP}

$\mathrm{Nil}$

\section{ETHICAL CONSIDERATION}

Ethical clearance was obtained from Institutional Ethical Committee of AIMS and Research Committee of Amrita College of Nursing

\section{AUTHORS CONTRIBUTION}

Bency Bhasy conceived of the presented the idea. Lekha Viswanath designed the conceptual frame work and tool. Linda Varghese and Aswathy Krishnan verified the analytical methods and whole manuscript. Bency Bhasy wrote the manuscript in consultation with Linda Varghese and Aswathy Krishnan.

\section{CONFLICTS OF INTEREST}

There are no conflicts of interest.

\section{REFERENCES}

1. Senthilkumar N, Anandhasayanam A, Safil KM, Shemimol S. A comparative study on the effectiveness of Angiotensin Converting Enzyme Inhibitors (ACEIS) and Angiotensin Receptor Blockers (ARBS) in Diabetic Nephropathy in Type 2 Diabetes Mellitus patients. Int J Pharm Pharm Sci 2016;8:289-92.

2. De Chesnay M, Anderson BA. Caring for the Vulnerable Patients: Perspectives in Nursing Theory, Practice and Research. $4^{\text {th }}$ ed., Vol. 2. Burlington: Jones and Bartlett; 2016. p. 150.

3. de Holanda1 VR, de Souza MA, dos Santos Rodrigues MC, Bezerra Pinheiro AK, Coelho Damasceno MM. Knowledge of pregnant women 
about Gestational Diabetes Mellitus. J Nurs Port 2012;24:17-27.

4. Reder AV, Martin LL, Koniak-Griffin D. Maternity Nursing, Family, Newborn, and Women's Health care. 19 ${ }^{\text {th }}$ ed., Vol. 2. Thrissur: Lippincott William \& Wilkins Publishers; 2014. p. 142-6.

5. Sreedevi A, Gopalakrishnan UA, Ramaiyer SK, Kamalamma L. A randomized controlled trial of the effect of yoga and peer support on glycaemic outcomes in women with Type 2 diabetes mellitus: A feasibility study. BMC Complement Altern Med 2017;17:152-64.

6. James D, Steer PJ, Weiner CP, Gonik B. High Risk Pregnancy and Management Option. $3^{\text {rd }}$ ed., Vol. 8. Nottingham, UK: British Library; 2001. p. 291-305
7. Jayakrishnapillai PV, Nair SV, Kamalasanan K. Current trend in drug delivery considerations for subcutaneous insulin depots to treat diabetes. Colloids Surf B Biointerfaces 2017;153:123-31.

8. Ali SH, Al-nuaimi AM, Al-musawi BJ. Serum irisin and leptin levels in obese and non-obese women with polycystic ovary syndrome with reference to glucose homeostasis. Int J Pharm Pharm Sci 2016;8:276-83.

9. Zera CA, Nicklas JM, Levkoff SE, Sc D, Seely EW. Diabetes risk perception in women with recent gestational diabetes: Delivery to the postpartum visit. Matern Fetal Neonatal Med 2013;26:691-6.

10. Amason JS. The effect of an educational intervention in women with gestational diabetes: A pilot study. Georgia State Univ 2013;1:23-48. 